\title{
Portable Automated Radio-Frequency Scanner for Non-destructive Testing of Carbon-Fibre-Reinforced Polymer Composites
}

\author{
B. Salski ${ }^{1}$ - W. Gwarek ${ }^{1}$ - P. Kopyt ${ }^{1}$ - P. Theodorakeas ${ }^{2}$ - I. Hatziioannidis ${ }^{2}$. \\ M. Koui ${ }^{2}$ - A. Y. B. Chong ${ }^{3}$ - S. M. Tan ${ }^{3}$ - V. Kappatos ${ }^{3}$ - C. Selcuk ${ }^{3}$ - T. H. Gan ${ }^{3}$
}

Received: 7 August 2015 / Accepted: 25 February 2016 / Published online: 28 March 2016

(C) The Author(s) 2016. This article is published with open access at Springerlink.com

\begin{abstract}
A portable automated scanner for non-destructive testing of carbon-fibre-reinforced polymer (CFRP) composites has been developed. Measurement head has been equipped with an array of newly developed radio-frequency (RF) inductive sensors mounted on a flexible arm, which allows the measurement of curved CFRP samples. The scanner is also equipped with vacuum sucks providing mechanical stability. RF sensors operate in a frequency range spanning from 10 up to $300 \mathrm{MHz}$, where the largest sensitivity to defects buried below the front CFRP surface is expected. Unlike to ultrasonic testing, which will be used for reference, the proposed technique does not require additional couplants. Moreover, negligible cost and high repeatability of inductive sensors allows developing large scanning arrays, thus, substantially speeding up the measurements of large surfaces. The objective will be to present the results of an extensive measurement campaign undertaken for both planar and curved large CFRP samples, pointing out major achievements and potential challenges that still have to be addressed.
\end{abstract}

Keywords CFRP · Scanner · Inductive sensors

B. Salski

bsalski@ire.pw.edu.pl

1 Institute of Radioelectronics and Multimedia Technology, Warsaw University of Technology, Warsaw, Poland

2 Materials Science \& Engineering Department, National Technical University of Athens, Athens, Greece

3 Brunel Innovation Centre, Brunel University London, Middlesex, UK

\section{Introduction}

Future generation of engineered structures in the civil, aerospace, automotive and marine industries will consist mostly of carbon composite materials, due to their performance and structural efficiency. However, one of major issues to be resolved is that the modes of failure in composite intensive structures such as the increasingly popular carbon-fibrereinforced polymer (CFRP), composite reinforced concrete beams [1-3] or the recent operational aircraft, Boeing 787 Dreamliner (with $50 \%$ composite) [4], are not fully known as they are still near the beginning of their design life. It is clear that these CFRP materials are susceptible to internal impact damage, not visible with an un-aided eye at the surface. In spite of this, inspections at the point of manufacture and in service is largely manual with consequent low area coverage. Operation downtime is usually inevitable during scheduled or unscheduled inspection. Common non-destructive testing (NDT) techniques utilized for CFRP include ultrasonic testing (UT) [5,6], eddy current testing (ECT) [7-9], shearography [10], microwave and millimeter wave characterization $[11,12]$. However, results obtained are difficult to interpret for most NDT techniques due to the intrinsic anisotropy and inhomogeneity of the CFRP structure [13]. Moreover, there are requirements for specific techniques which may be easily applicable in-situ.

One of promising techniques that successfully addresses the aforementioned challenges is radio-frequency inductive testing (RFIT) $[14,15]$ with a single sensor built of two coupled spiral inductors manufactured on a printed circuit board (PCB). First of all, as it has been shown in [14], the point spread function (PSF) of the sensor is strongly anisotropic, thus, enabling the characterization of such materials like CFRP. Second of all, the measurement with the RFIT sensor allows easily determining the depth of defects buried 
in the CFRP materials and obtain a cross-sectional scan by the appropriate combination of measurements data at a few frequencies. The RFIT technique does not require calibration as the obtained RF C-scan is a differential measure of the magnitude of power transmission coefficient. In addition, RFIT sensors can be developed on PCB with a very high repeatability, thus, substantially suppressing any issues associated with the use of large scanning arrays.

The RFIT technique is similar to ECT $[9,14]$, as both methods take the advantage of electromagnetic fields to sense the material under test (MUT). However, there are a few differences thoroughly pointed out in [14]. First of all, RFIT is not necessarily based on eddy currents sensing, but rather on surface currents induced in the vicinity of the gap between adjacent inductors, which gives more flexibility in adjusting sensor's characteristics to the given properties of the MUT. For instance, induction of typical coils wounded on a ferrite core applicable in ECT drops at frequencies higher than a couple of $\mathrm{MHz}$, mainly due to increasing capacitive parasitics and magnetic loss of ferrites. On the contrary, simple planar inductors manufactured on a printed circuit board, which do not exhibit any frequency limitations in RF spectra, are used in the RFIT technique. Moreover, magnitude of power transmission measured in that technique allows achieving sensitivity to buried defects at the level of $3 \mathrm{~dB}$, which means $100 \%$ change of transmitted power with respect to the measurement over the area without defects.

As a result of the recent successful development of the planar coupled spiral inductors tailored for the NDT of CFRP composites $[14,15]$, the sensor and auxiliary electronic modules were subject to further development [16] in which a line array of sensors is realized. The sensor array is integrated with a portable automated scanner. Since the sensors and measurement procedures have already been discussed in [14-16], the attention is focused in this paper on the scanner developed to carry out the experiments validating the whole RFIT NDT system on both planar and curved CFRP composites with typical buried and surface defects. The results clearly demonstrate the applicability of such automated RFIT system for reliable and efficient CFRP inspection.

\section{Scanner}

The whole NDT system, as shown in Fig. 1, consists of the $\mathrm{XY}$ scanner with a sensor array, data acquisition board (DAQ), and a PC station.

\subsection{XY Scanner}

One of major objectives was to minimize a scanner's weight, while providing high scanning rates, good resolution, positioning repeatability in both absolute and relative

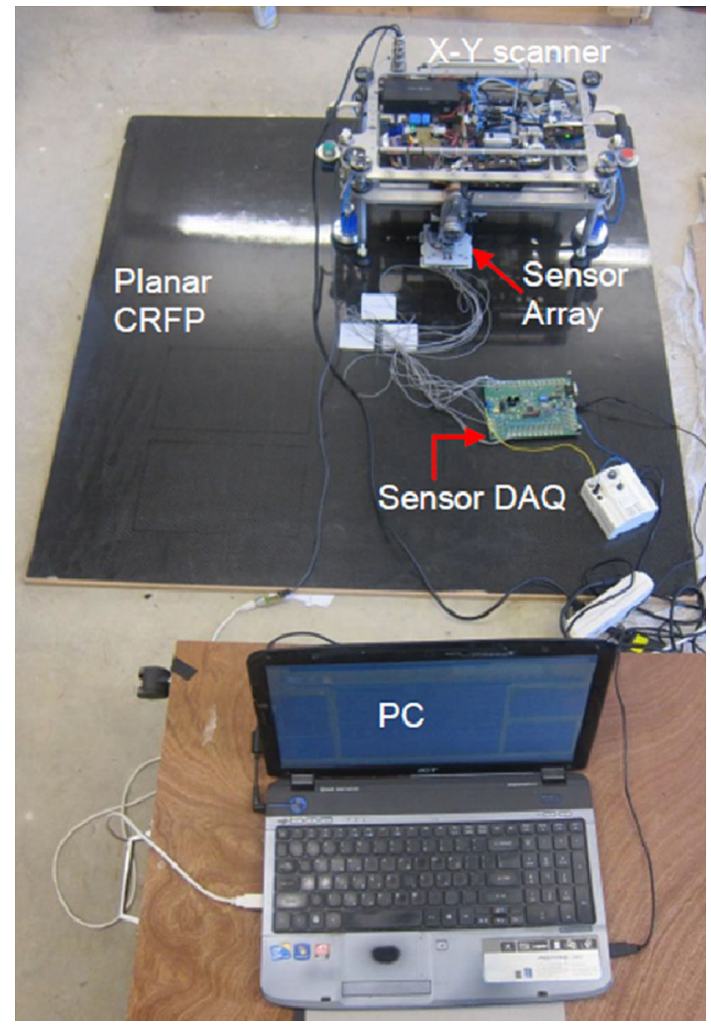

Fig. 1 RF NDT scanner setup

coordination systems, involving the minimum degrees of freedom. For that reason, an important functional requirement was the development of an ergonomic portable chassis with the weight less than $10 \mathrm{~kg}$. Furthermore, the necessary space for seamless integration of all modalities that will implement the requested functional requirements of the scanner should be provided. Based on the aforesaid, the scanner frame was designed and manufactured through the use of two aluminum plates as presented on a schematic design in Fig. 2. The chassis was secured via the application of four tubes with collars that interconnect the two layers of aluminum plates. The aluminum plates were pre-cut with all necessary cut-outs and bores so as to be able to attach all necessary parts, gantry, motor supports, electronics, and pneumatic systems. The chassis structure has the advantage that all parts are accessible and can be removed readily for repair or replacement. It can be easily disassembled, machined if modifications are necessary during service period and reproduced on request. The overall size of the chassis frame is $600 \times 400 \times 250 \mathrm{~mm}^{3}$.

The main function of the $x-y$ gantry system is to implement a scanning motion of a sensor arm. For that purpose, an Igus DryLin stage drive was selected, which provides lubricant-free linear axles that are driven either by trapezoidal thread, steep thread or toothed belt. The user can choose a suitable individual solution from lightweight solid plastic units up to massive stainless steel solutions. For our appli- 


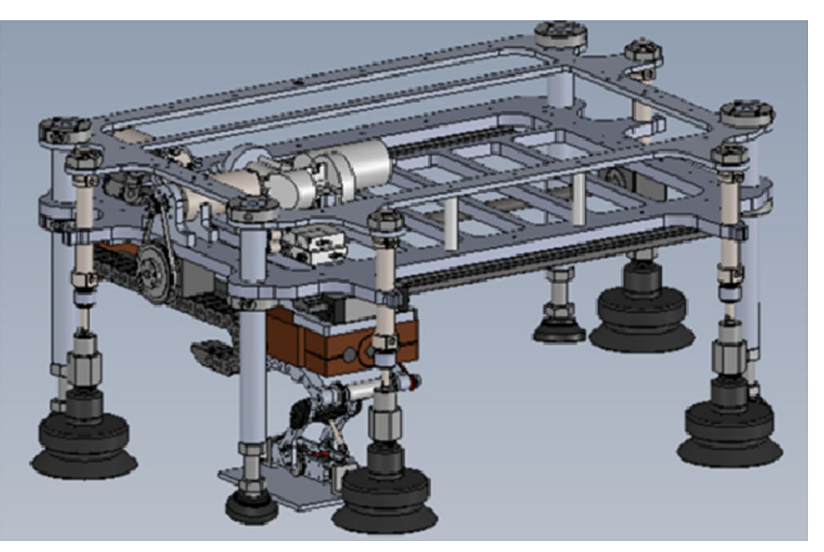

Fig. 2 Scanner platform with XY precision translators, sensor arm and vacuum sucks

cation trapezoidal threads with $2 \mathrm{~mm}$ pitch were chosen for both $\mathrm{X}$ and $\mathrm{Y}$ directions. Along with robust design of these components, their main features include ruggedness and insensitivity to dirt, water, chemicals, heat or impacts.

Magnetic encoders have a resolution of 1024 ticks per shaft revolution. One revolution is converted to a linear motion of $2 \mathrm{~mm}$ with a resolution of $10 \mu \mathrm{m}$ per revolution of the DC motors. The power source is mounted within the chassis boundaries and provides the scanner with voltage supply options of 5-12-24 VDC. The scanner is controlled via RS485 protocol converted to USB before it is connected to the PC.

Another function of the scanner is the attachment to the CFRP surface. Due to operational principles of the inspection method and the composite material characteristics, it is not possible to implement magnets and clamping systems for the robust mounting of the scanner. For that reason, plastic suction cups were developed, which satisfy two crucial conditions: (a) there is no magnetic field interference with RF sensors, and (b) no surface damage can be induced to the MUT. The mounting of the scanner is performed by the following procedure. Initially, the operator places the scanner on the MUT's surface, ensuring that the supporting legs and the plastic cups are conformed on the surface. Subsequently, pressure can be produced to the cups by pressing an air compressor activation button. As a result of a network pressure of 8 bar, the scanner becomes firmly attached to the surface.

\subsection{Sensor Arm}

Sensor arm, as shown in Fig. 3, is a mounting platform for the array of RF sensors. It is made of aluminum housed with bearings, pivot brackets, and springs to produce a lab jack mechanism with better conformance of the sensors to the MUT's surface. RF sensors are placed in PA6 plastic protection pads, which are mounted to a holder arm with the aid of a plastic holder plate to prevent any large metallic parts in the

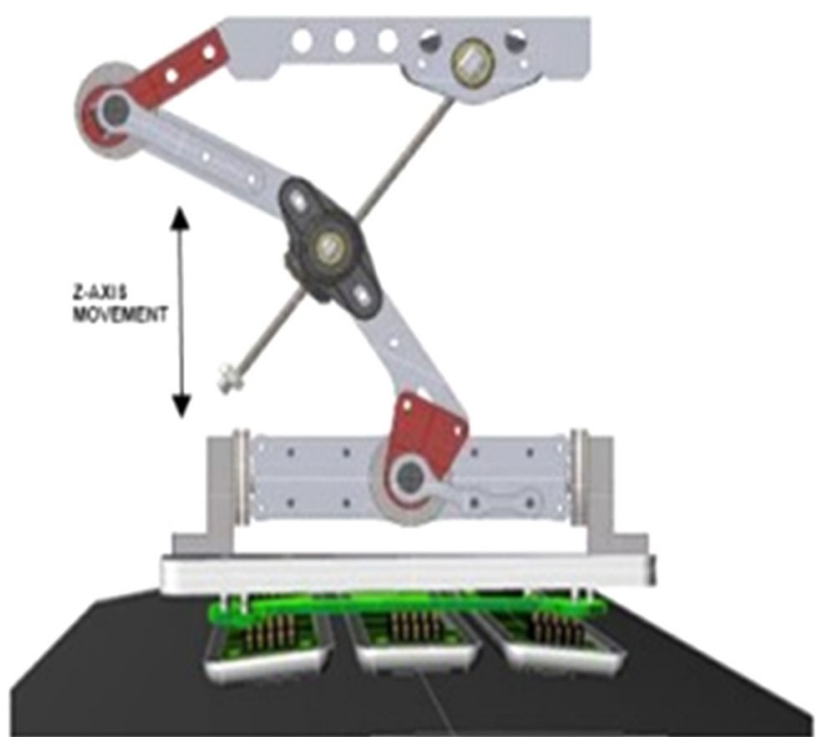

Fig. 3 Sensor flexible arm

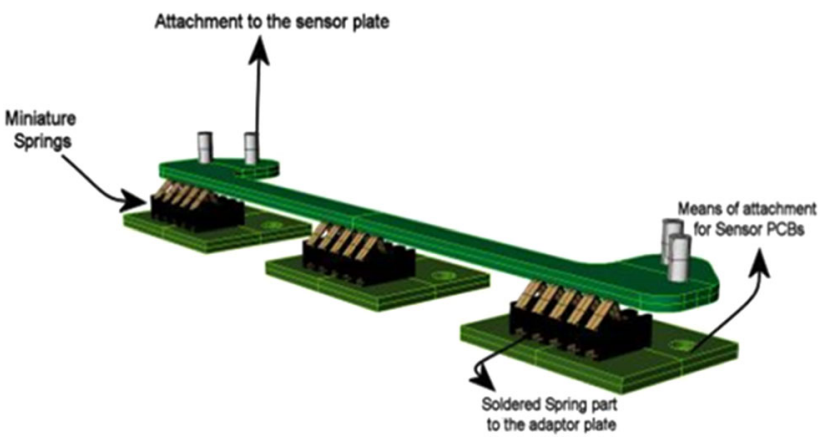

Fig. 4 Mounting pad of RF sensors

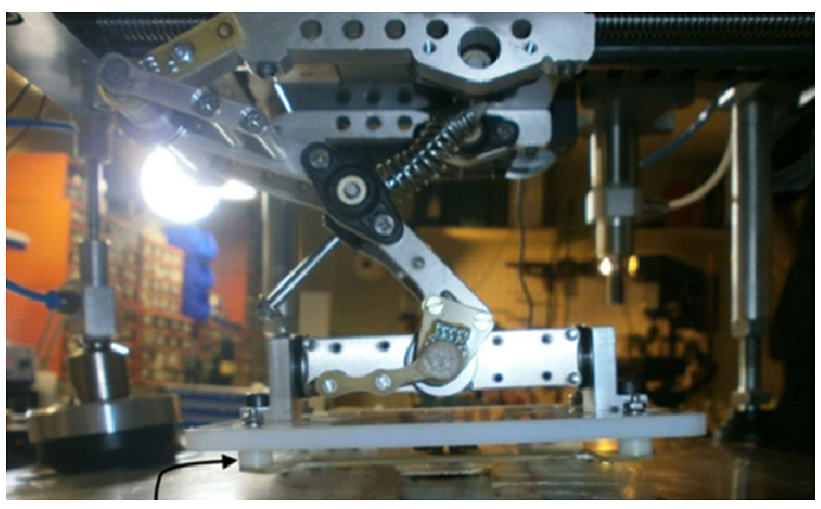

Fig. 5 Photograph of a sensor arm

vicinity of the sensors. Although mounting pads indicated in Fig. 4 fit precisely to the plastic protection pads, the attachment can be further enhanced with thermoplastic adhesive. The dimensions of the holder plate are $100 \times 120 \mathrm{~mm}^{2}$ with a thickness of $8 \mathrm{~mm}$ (see Fig. 5). 

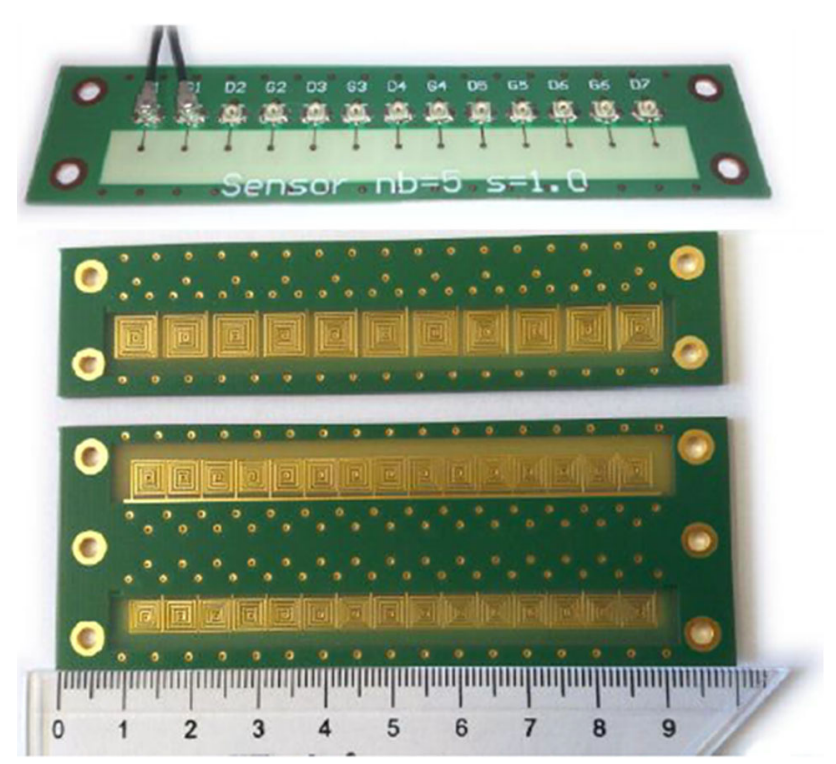

Fig. 6 Exemplary RF sensor line and array

Vertical force pushing the sensors to the CFRP surface is provided by the lab jack mechanism that drives a passive spring on the outside of a telescopic pair shown in Fig. 4. An exerted force over the sensor plate is adjusted by the use of springs in order to maintain a firm contact with the MUT's surface during a sliding movement of the sensors. The total travel distance on the $\mathrm{z}$-axis is $40 \mathrm{~mm}$, enabling the application of proper pressure to the sensor plate, in order to ensure its smooth conformance on curved surfaces. The vertical displacement can be monitored with an integrated absolute encoder.

\subsection{RF Sensors}

Sensor array is made in the form of a line of RF inductors manufactured on the PCB, although arrays are also developed and used, as shown in Fig. 6. Single measurement utilizes two adjacent inductors treated as primary and secondary windings of a transformer. As a result, there are $N-1$ measurement points for $N$ aligned inductors [16].

If RF sensors are attached to the MUT's surface with the aid of the sensor arm, a magnetic field penetrates the MUT provided that the penetration depth $d_{p}$ is large enough at a given frequency. For instance, conductivity of CFRP composites is usually at the level of about $\sigma=10^{4} \mathrm{~S} / \mathrm{m}$ or more $[13,14]$, which means $d_{p}$ is over $1 \mathrm{~mm}$ for frequencies below ca. $30 \mathrm{MHz}$. It implicitly determines the frequency range for RF inductors if the thickness of CFRP panels is given.

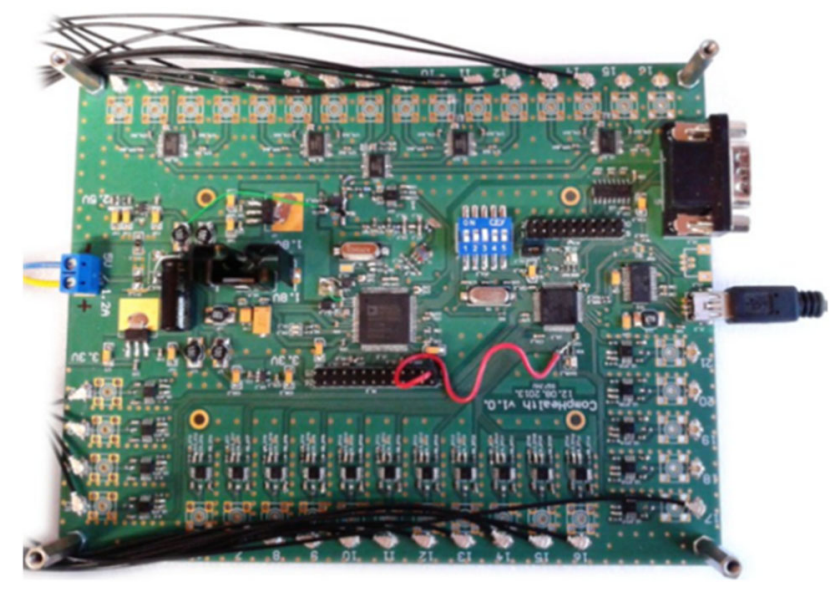

Fig. 7 Data acquisition board

\subsection{Data Acquisition Board}

In the view of the above considerations of the frequency range of operation of the RF sensors, electronic circuitry, dedicated to the measurement of the number of individual sensor channels, has been developed (see Fig. 7). The main role in that system is played by a direct digital synthesizer (DDS), controlled with a 32-bit ST microcontroller, which also serves as an interface with a PC host station [16]. DDS generates signals spanning from $20 \mathrm{MHz}$ up to $300 \mathrm{MHz}$, as this is the spectrum where the largest sensitivity to defects buried in ca. $1 \mathrm{~mm}$ thick CFRP panels is expected. The signal is multiplexed sequentially to all measurement channels. Each channel is equipped with an individual logarithmic wideband power detector, which is to be connected to the secondary winding of a given RF sensor. As a result, each measurement shot, consisting of frequency sweeping, provides the whole spectrum of power transmission through each coupled sensor pair, as indicated in Fig. 6. The system does not require any calibration procedures to be invoked as the whole RF image can be studied using comparative measures (see details in [14]).

\section{Measurements}

Characterization of flat and curved CFRP panels will be presented in this Section. Both panels, manufactured by ATARD [17], consist of four layers of CFRP twill immersed in epoxy resin. All the samples have been measured with a single line of RF inductive sensors shown at the top of Fig. 6 mounted in the scanner depicted in Fig. 1 with $N_{a}=12$ measurement pairs. As the scanning step has been set to $2 \mathrm{~mm}$, an image of a $300 \times 200 \mathrm{~mm}^{2}$ surface consists of over 15,000 points, which means that the scanner has to undertake 1250 incremental shifts across the surface. That number can 


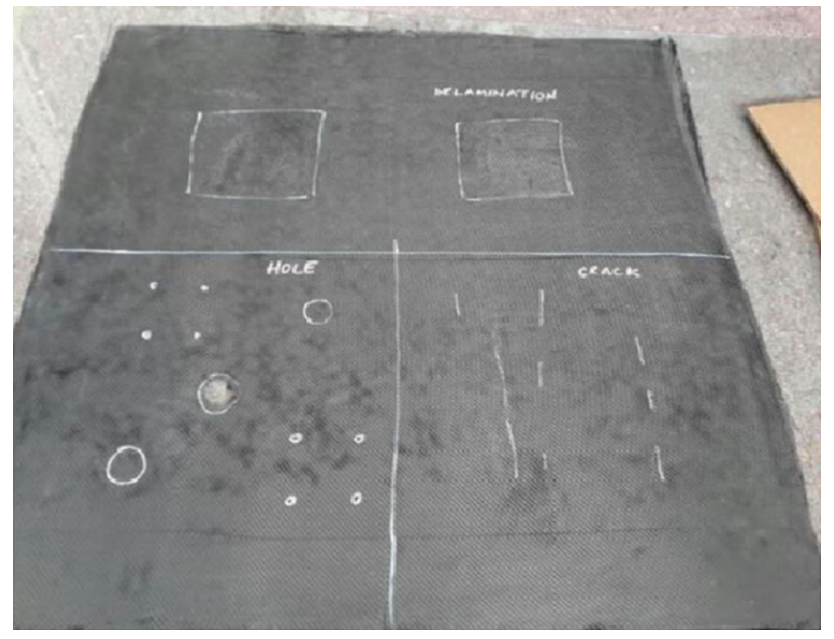

Fig. 8 Flat CFRP panel

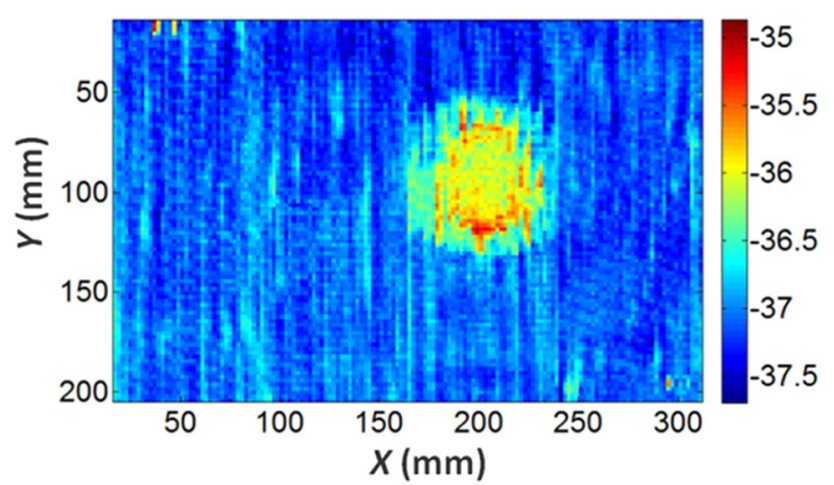

(a)

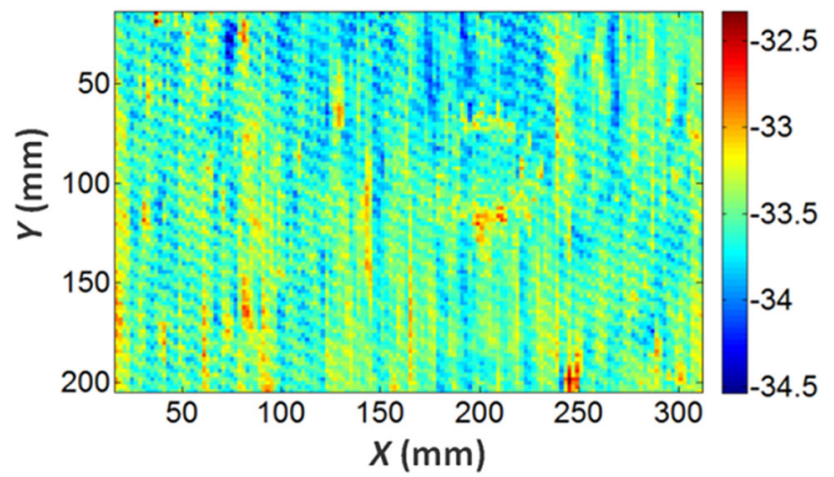

(b)

Fig. 9 Hole buried in the CFRP sample shown in Fig. 8 measured at a $50 \mathrm{MHz}$ and b $200 \mathrm{MHz}$

be further decreased if additional sensor lines are exploited in the scanner (see Fig. 3), which will be the subject for future enhancements of the system.

A single measurement of an individual sensor at a given frequency takes about $t_{e}=8 \mu \mathrm{s}$, while each mechanical shift to another position lasts for ca. $t_{m}=0.5 \mathrm{~s}$. Hence, the scanning

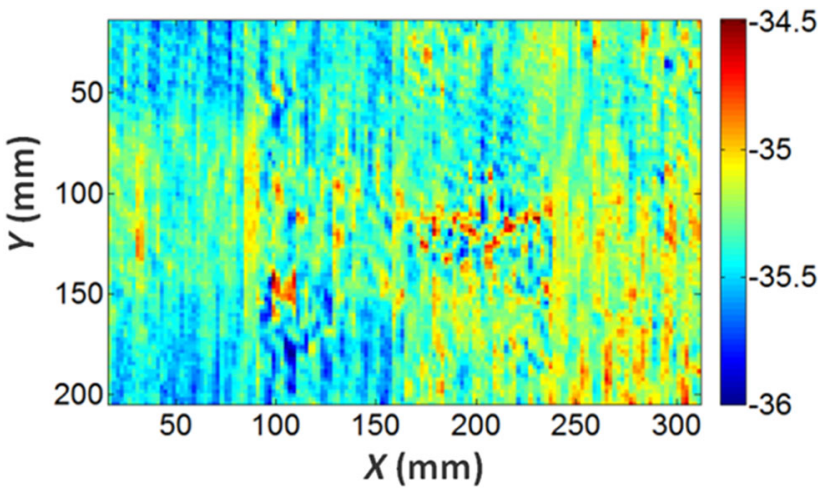

Fig. 10 Specific bubbles buried in the CFRP sample shown in Fig. 8 measured at $20 \mathrm{MHz}$

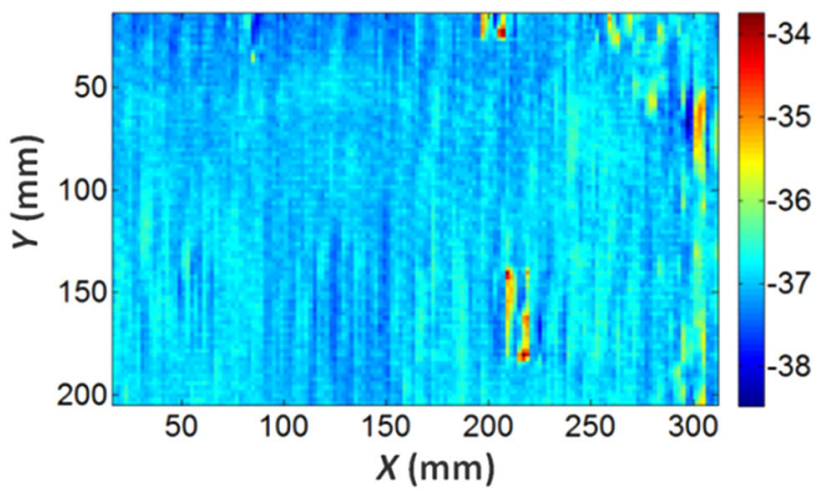

(a)

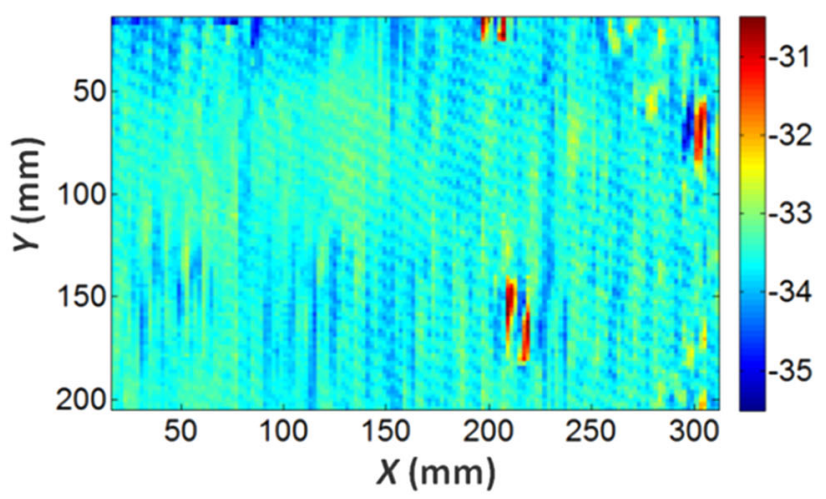

(b)

Fig. 11 Cracks buried in the CFRP sample shown in Fig. 8 measured at a $20 \mathrm{MHz}$ and b $300 \mathrm{MHz}$

rate at $N_{f}=10$ frequencies with the array shown in Fig. 6 reaches about

$R T=\frac{N_{f} N_{a}}{N_{f} N_{a} t_{e}+t_{m}} \cong 82$

measurement shots per second. However, the increase of the sensor array from one to four lines $\left(N_{a}=48\right)$ would augment RT up to 110 , by reducing the deteriorating contribution of the mechanical shift on the overall inspection time. There is 


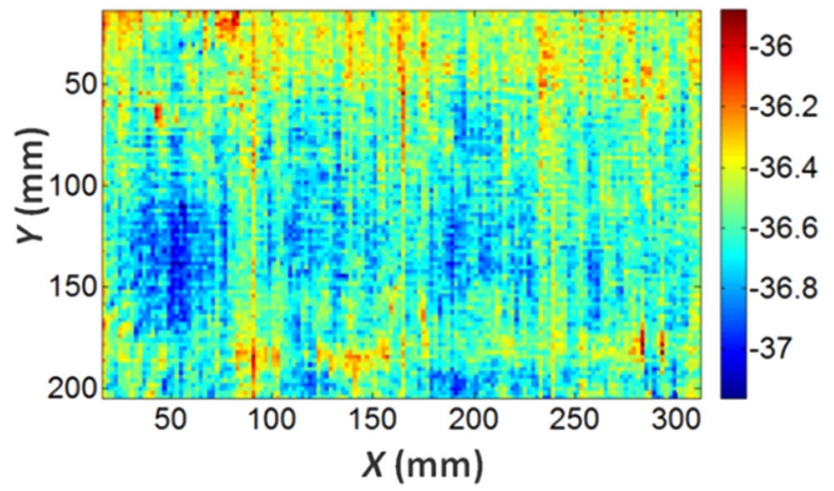

(a)

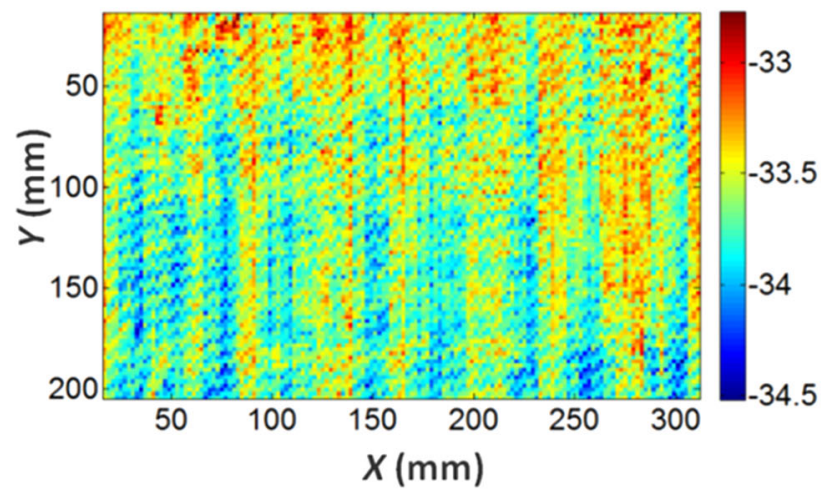

(b)

Fig. 12 Delamination buried in the CFRP sample shown in Fig. 8 measured at a $20 \mathrm{MHz}$ and b $300 \mathrm{MHz}$

still much room for further improvements of the scanning rate by the reduction of $t_{e}$, e.g. by exchanging sequential data acquisition with a parallel one, which may speed up the measurements by the order of magnitude or more.

Although the measurement step is as large as $2 \mathrm{~mm}$, a large area of the PSF of a single RF sensor allows detecting even strong localized spots (see [14] for details). The knowledge on the spatial distribution of the PSF can be used to enhance the RF image resolution much further, but that issue goes beyond the scope of this paper.

\subsection{Flat CFRP Panel}

A flat CFRP panel is shown in Fig. 8. As it can be noticed, a few types of defects have been intentionally introduced, such as holes, bubbles, cracks, and delamination. As in [14], all $\mathrm{RF}$ images presented hereafter are constructed from a power transmission coefficient given in a logarithmic dB-scaling. Figure 9 shows RF images stored at two distinct frequencies at the south west part of the panel shown in Fig. 8. The image at $50 \mathrm{MHz}$ clearly indicates a large hole with the diameter of ca. $55 \mathrm{~mm}$, which is not visible with an unaided eye at the front surface of the panel. It is confirmed by the lack

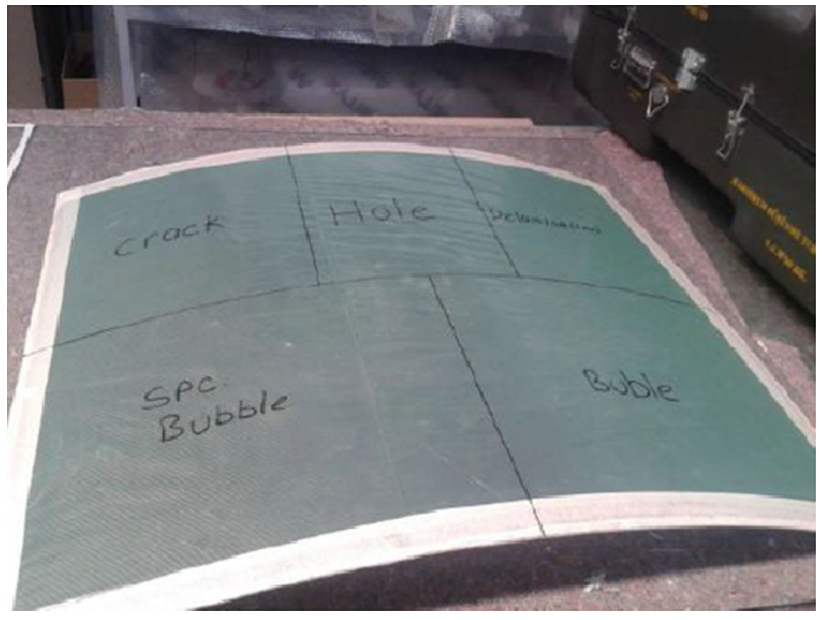

Fig. 13 Curved CFRP panel

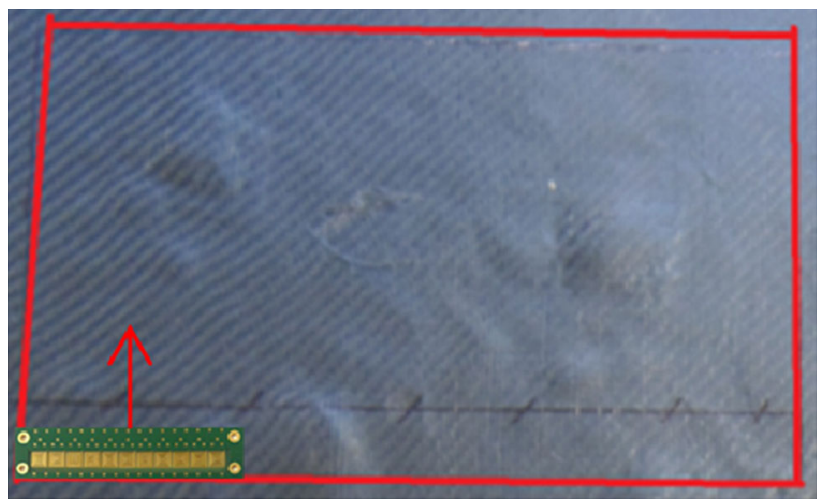

Fig. 14 Specific bubbles on the curved CFRP sample shown in Fig. 13

of distinctiveness of the hole at the RF image measured at $200 \mathrm{MHz}$, which can be attributed to a substantially smaller penetration depth at this frequency.

In particular, regular fracture appearing in Fig. $9 \mathrm{~b}$ is correlated with the orientation of the front CFRP twill [15]. Moreover, the RF image measured at a higher frequency of $200 \mathrm{MHz}$, which is more sensitive to surface roughness of the inhomogeneous CFRP sample, contributes to larger fluctuations visible in Fig. 9b. In addition, scanner arm and other mechanisms controlling the movement of the sensors introduce additional uncertainty, thus, leading to irregular features of RF images.

Figure 10 indicates an RF image measured at $20 \mathrm{MHz}$ at the areas of the flat CFRP panel shown in Fig. 8, where buried bubbles were intentionally introduced by the manufacturer. Indeed, several tiny fluctuations can be observed in the central part and the right-hand side of the image, while the changes at the left-hand side of the image are rather smooth and results mostly due to slight misalignments of the CFRP twill.

Subsequently, Fig. 11 shows RF images stored at the south east part of the panel shown in Fig. 8 with vertical cracks 


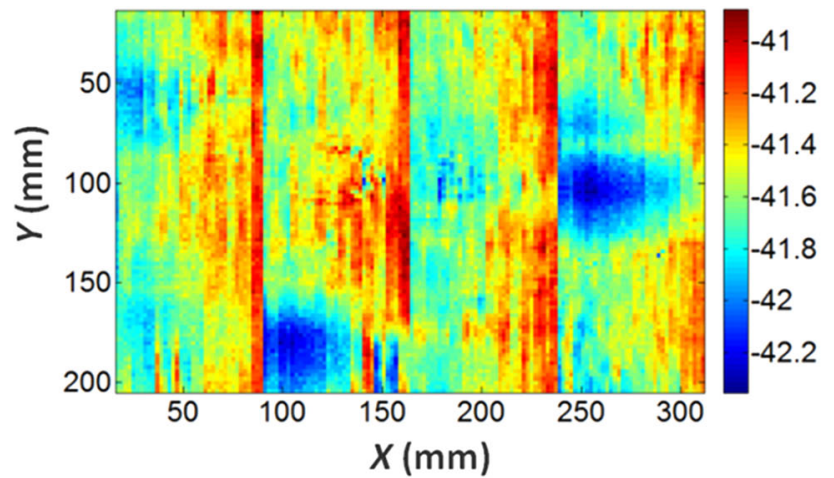

(a)

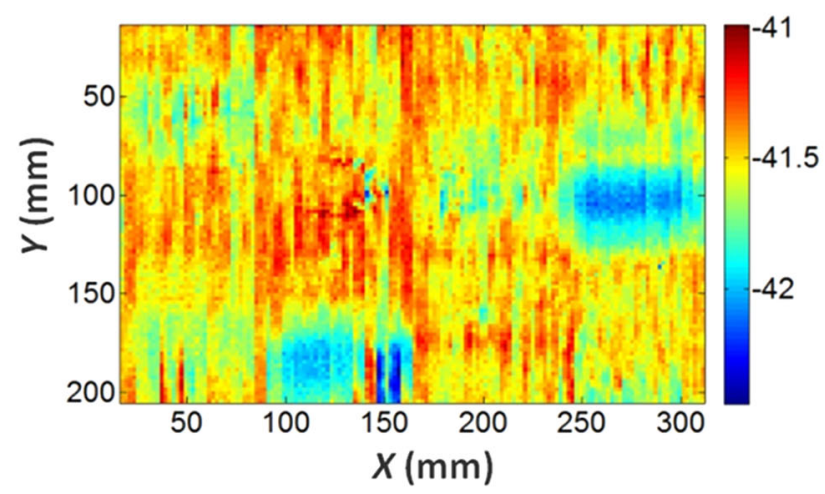

(b)

Fig. 15 Specific bubbles on the CFRP sample shown in Fig. 14 and measured at $20 \mathrm{MHz}$. a Raw data. b Linear lift off removed

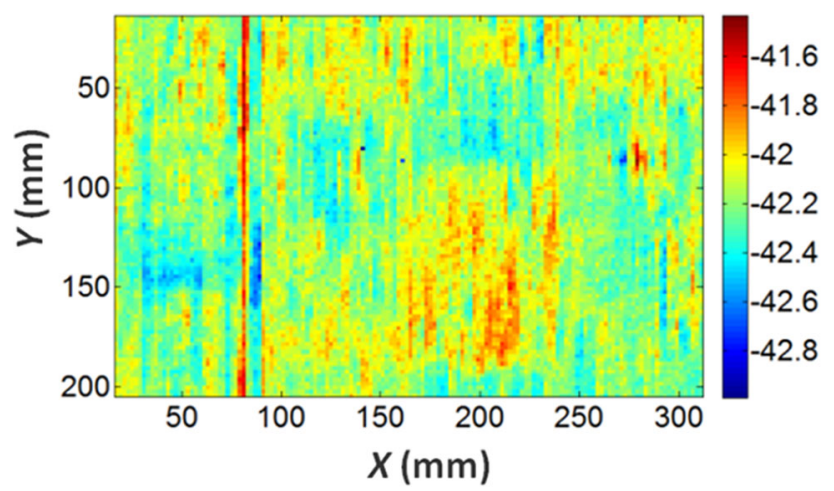

Fig. 16 Delamination buried in the CFRP sample shown in Fig. 14 measured at $20 \mathrm{MHz}$. Linear lift off is removed

buried at selected regions of the CFRP sample. As the cracks are visible even at $300 \mathrm{MHz}$, it can be concluded that the defects are spanning almost across the whole thickness of the sample, although those are not visible with an unaided eye. The length of the cracks visible in the middle of the RF images is about $40 \mathrm{~mm}$.

Eventually, Fig. 12 shows delamination buried in the CFRP sample shown in Fig. 8. As it can be noticed in Fig. 12a, there is large deep minimum at a left-hand side of the

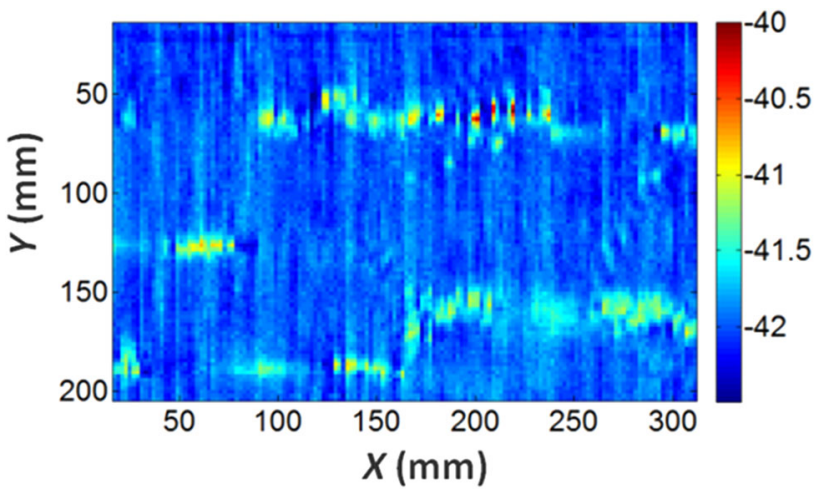

Fig. 17 Cracks buried in the CFRP sample shown in Fig. 14 measured at $20 \mathrm{MHz}$. Linear lift off is removed

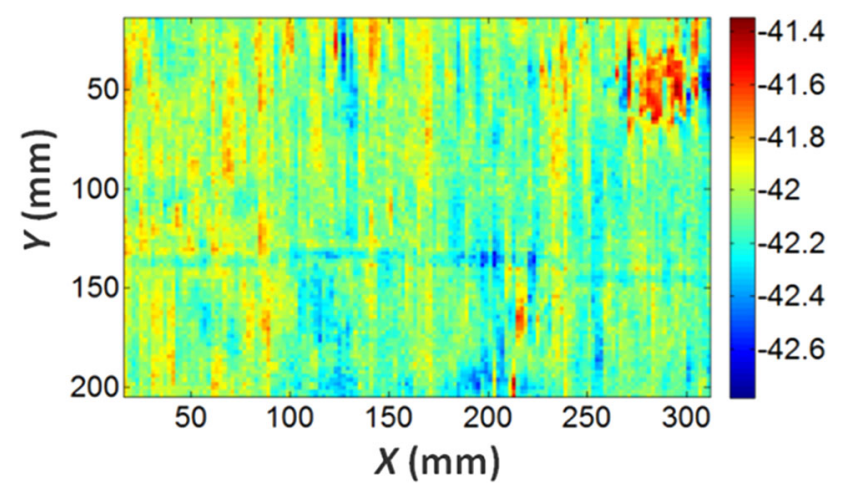

Fig. 18 Holes buried in the CFRP sample shown in Fig. 14 measured at $20 \mathrm{MHz}$. Linear lift off is removed

image, where the defect is expected and which cannot be noticed at $300 \mathrm{MHz}$, what indicates its deeper location.

\subsection{Curved CFRP Panel}

Figure 13 shows the sample of a curved CFRP panel with several types of defects introduced in a similar way as in the flat sample. Curvature radius of the panels is ca. $1.5 \mathrm{~m}$. As it will be shown, measurement of curved panels brings additional issues related to uneven attachment of the sensor line to the surface (lift off), which has to be de-embedded from raw measurement data.

Figure 14 depicts specific bubbles occurring at a front surface of the curved CFRP panel. Due to the chosen direction of the sensor indicated in Fig. 14, the sensor's line in unevenly attached to the curved surface and, consequently, the corresponding RF image consists of four individual stripes, as show in in Fig. 15a. Fortunately, the uneven attachment results in a linear lift off occurring in power transmission measured along the sensor line, so it can be determined and removed at a post-processing stage. Consequently, Fig. 15b is smoother enabling better recognition of the bubbles, the location of which corresponds very well with those shown in Fig. 14. 
Figures 16,17,18 show another types of defects measured with the RF scanner at $20 \mathrm{MHz}$ with the linear trend already removed. Figure 16 indicates the RF image stored in the area where delamination is expected and, indeed, it can be seen in the middle of the image. In addition to that, there is also a vertical defect visible at the left-hand side of the RF image shown in Fig. 16, which may be some buried crack unintentionally introduced by the manufacturer.

Subsequently, Fig. 17 shows several horizontal cracks measured on the curved CFRP sample. Eventually, Fig. 18 depicts the hole clearly visible in the top right corner of the image. Similarly to the unintentional crack depicted in Fig. 16, there is a horizontal fault visible in Fig. 18, which has not been explicitly highlighted by the manufacturer.

Acknowledgments Part of this work has received funding from EU 7th Framework Programme managed by REA under grant agreement no. 314935 and from the Polish National Ministry of Science and Higher Education under grant agreement no. 2826/7.PR/2013/2. The research has been undertaken as a part of the project entitled "Radio Frequency Sensing for Non-Destructive Testing of Carbon Fibre Reinforced Composite Materials for Structural Health Monitoring"-Comp-Health, in the collaboration between the following organizations: E.T.S. Sistemi Industriali srl, Kingston Computer Consultancy Limited, Nemetschek ood, Atard a.s., UAB Elmika, Warsaw University of Technology, National Technical University of Athens and Brunel Innovation Centre of Brunel University London.

Open Access This article is distributed under the terms of the Creative Commons Attribution 4.0 International License (http://creativecomm ons.org/licenses/by/4.0/), which permits unrestricted use, distribution, and reproduction in any medium, provided you give appropriate credit to the original author(s) and the source, provide a link to the Creative Commons license, and indicate if changes were made.

\section{References}

1. Zhou, Y., Guo, M., Zhang, F., Zhang, S., Wang, D.: Reinforced concrete beams strengthened with carbon fibre reinforced polymer by friction hybrid bond technique: Experimental investigation. Mater. Des. 50, 130-139 (2013)

2. Garcia, R., Helal, Y., Pilakoutas, K.: Bond behavior of substandard splices in RC beam externally confined with CFRP. Constr. Build. Mater. 50, 340-351 (2014)

3. Zhao, X.L., Zhang, L.: State-of-the-art review on FRP strengthened steel structures. Eng. Struct. 26(8), 1808-1823 (2007)

4. Boeing: www.compositesworld.com/articles/boeing-787-update. Accessed 05 Jan 2016

5. Scarponi, C., Briotti, G.: Ultrasonic technique for the evaluation of delaminations on CFRP, GFRP, KFRP composite materials. Compos. B Eng. 31(3), 237-243 (2000)
6. Farinas, M.D., Alvarez-Arenas, T.E.G., Aguado, E.C., Merino, M.G.: Non-Contact ultrasonic inspection of CFRP prepregs for aeronautical applications during lay-up fabrication. In: IEEE IUS Book Series, pp. 1586-1589 (2013)

7. Cheng, J., Ji, H.L., Qiu, J.H., Takagi, T., Uchimoto, T., Hu, N.: Novel electromagnetic modeling approach of carbon fibrereinforced polymer laminate for calculation of eddy currents and eddy current testing signals. J. Compos. Mater. 49(5), 617-631 (2015)

8. Mook, G., Lange, R., Koeser, O.: Non-destructive characterization of carbon-fibre-reinforced plastics by means of eddy-currents. Compos. Sci. Technol. 61(6), 865-873 (2001)

9. Heuer, H., Schulze, M., Pooch, M., Gabler, S., Nocke, A., Bardl, G., Cherif, Ch., Klein, M., Kupke, R., Vetter, R., Lenz, F., Kliem, M., Bulow, C., Goyvaerts, J., Mayer, T., Petrenz, S.: Review on quality assurance along the CFRP value chain-nondestructive testing of fabrics, preforms and CFRP by HF radio wave techniques. Compos. B 77, 494-501 (2015)

10. Taillade, F., Quiertant, M., Benzarti, K.: Shearography and pulsed stimulated infrared thermography applied to a nondestructive evaluation of FRP strengthening systems bonded on concrete structures. Constr. Build. Mater. 25, 568-574 (2011)

11. Ravuri, M., Abou-Khousa, M., Kharkovsky, S., Zoughi, R., Austin, R.: Microwave and millimeter wave near-field methods for evaluation of radome composites. Rev. Prog. Quant. Nondestr. Eval. Quant. Nondestr. Eval. 27B, 976-981 (2008)

12. Kharkovsky, S., Zoughi, R.: Microwave and millimeter wave nondestructive testing and evaluation-overview and recent advances. IEEE Instrum. Meas. Mag. 10(20), 26-38 (2007)

13. Cheng, J., Ji, H.L., Qiu, J., Takagi, T., Uchimoto, T., Hu, N.: Role of interlaminar interface on bulk conductivity and electrical anisotropy of CFRP laminates measured by eddy current method. NDT\&E Int. 68, 1-12 (2014)

14. Salski, B., Gwarek, W., Korpas, P.: Electromagnetic inspection of carbon-fiber-reinforced polymer composites with coupled spiral inductors. IEEE Trans. Microw. Theory Technol. 63(7), 1535-1544 (2014)

15. Salski, B., Gwarek, W., Korpas, P., Reszewicz, S., Chong, A.Y.B., Theodorakeas, P., Hatziioannidis, I., Kappatos, V., Selcuk, C., Gan, T.H., Koui, M., Iwanowski, M., Zielinski, B.: Non-destructive testing of carbon-fibre-reinforced polymer materials with a radiofrequency inductive sensor. Compos. Struct. 122, 104-112 (2015)

16. Korpas, P., Salski, B., Reszewicz, S.: Multichannel measurement system for non-destructive testing of carbon-fiber-reinforced polymer composites. In: 20th International Conference on Microwaves, Radar and Wireless Communications, MIKON (2014)

17. ATARD: Defence and Aerospace Industry Advanced Technology Applications Research and Development Inc., private communication (2013) 